\title{
LAS DICTADURAS DE VARGAS Y FRANCO: IMPLICACIONES DE LA CONSAGRACIÓN DE LA MATERNIDAD PARA LA ENFERMERÍA
}

\author{
Tânia Cristina Franco Santos ${ }^{1}$, Ieda de Alencar Barreira², Antonio José de Almeida Filho ${ }^{3}$, Alexandre Barbosa \\ de Oliveira ${ }^{4}$
}

\footnotetext{
${ }^{1}$ Doutora em Enfermagem. Professora Adjunta da Escola de Enfermagem Anna Nery (EEAN) da Universidade Federal do Rio de Janeiro (UFRJ). Rio de Janeiro, Brasil. E-mail: taniacristinafsc@terra.com.br.

${ }^{2}$ Professora do Programa de Pós-Graduação da EEAN/UFRJ. Pesquisadora do CNPq. Rio de Janeiro, Brasil. E-mail: iedabarreira@openlink.com.br

${ }^{3}$ Doutor em Enfermagem. Professor Adjunto da EEAN/UFRJ. Rio de Janeiro, Brasil. E-mail: ajafilho@terra.com.br

${ }^{4}$ Doutorando do Programa de Pós-Graduação em Enfermagem da EEAN/UFRJ. Rio de Janeiro, Brasil. E-mail: alexbaroli@ yahoo.com.bt
}

\begin{abstract}
RESUMEN: Este estudio tuvo como objetivo describir las características históricas y sociales de Brasil y España en el contexto de los regímenes dictatoriales instaurados por Vargas y Franco, y analizar las representaciones sobre la maternidad en el contexto de esos regímenes y sus implicaciones para la enfermería. Es un estudio histórico y social cuyo método consideró como fuentes: documentos escritos brasileños y españoles. La recolección de los datos se hizó en el período de 13 de mayo a 29 de agosto de 2008. Para el análisis se realizaron las etapas de organización y clasificación de las fuentes elegidas. Como resultado, se evidencio que la glorificación de la mujer por sus virtudes físicas como la maternidad y por sus atributos morales legitimó su inserción honorable en el espacio público, para reproducir en ese espacio, como enfermeras, las cualidades femeninas necesarias al cuidado. Se concluye que la consagración de la maternidad ha determinado posibilidades y límites para la enfermera.
\end{abstract}

DESCRIPTORES: Enfermería. Historia de la enfermería. Enfermería obstétrica. Salud de la mujer.

\section{THE FRANCO AND VARGAS DICTATORSHIPS: IMPLICATIONS OF THE CONSECRATION OF MATERNITY FOR NURSING}

\begin{abstract}
This study aimed to describe the historical and social characteristics of Brazil and Spain within the context of the Vargas and Franco dictatorial regimes and analyze representations about maternity therein, as well as their implications for nursing. This historical and social study contemplated Brazilian and Spanish written documents method as its primary sources. Data was collected from May $13^{\text {th }}$ to August $29^{\text {th }}, 2008$. Data analysis included the organization and classification stages of the primary sources selected. As a result, it became clear that the glorification of women for their physical virtues, such as maternity, and for their moral attributes legitimated their honorable insertion in the public arena. Thus, as nurses, they were to reproduce the feminine qualities necessary for care in this same arena. The conclusion is the consecration of motherhood has determined possibilities and limits for nurse.
\end{abstract}

DESCRIPTORS: Nursing. History of nursing. Obstetrical nursing. Women’s healthy.

\section{AS DITADURAS DE VARGAS E FRANCO: IMPLICAÇÕES DA CONSAGRAÇÃO DA MATERNIDADE PARA A ENFERMAGEM}

\begin{abstract}
RESUMO: Este estudo teve como objetivo descrever as características histórico-sociais do Brasil e da Espanha no contexto dos regimes ditatoriais instaurados por Vargas e Franco e analisar as representações sobre a maternidade no contexto desses regimes e suas implicações para a enfermagem. Estudo histórico-social cujo método contemplou como fontes: documentos escritos brasileiros e espanhóis. A coleta dos dados foi realizada no período de 13 de maio a 29 de agosto de 2008. A análise comportou as etapas de organização e classificação das fontes selecionadas. Como resultado, evidenciou-se que a glorificação da mulher por suas virtudes físicas como a maternidade e por seus atributos morais legitimou sua inserção honrosa no espaço público, ainda que para reproduzir nesse espaço, como enfermeira, as qualidades femininas necessárias ao cuidado. Conclui-se que a consagração da maternidade determinou possibilidades e limites para a enfermeira.
\end{abstract}

DESCRITORES: Enfermagem. História da enfermagem. Enfermagem obstétrica. Saúde da mulher. 


\section{CONSIDERACIONES INICIALES}

Estado Novo en la historiografía brasileña representa el período dictatorial que, bajo la égida de Getúlio Vargas, tuvo inicio tras un golpe de estado en 10 de noviembre de 1937, vigorando hasta 29 de octubre de 1945. Cumple resaltar que Getúlio Vargas permaneció en el poder durante quince años, sucesivamente, inicialmente como jefe de un gobierno provisional (1930-1934), después, presidente electo por voto indirecto (1934-1937) e por fin, dictador (1937-1945), siendo depuesto en 1945.

La expresión Estado Novo fue empleada por la primera vez por Oliveira Salazar, en el inicio de la década de 1930, para justificar el régimen autoritario portugués. Su utilización algunos años después en Brasil, así como la incorporación a la Carta Constitucional de 1937 de dispositivos das constituciones de regímenes totalitarios de la época, llegó a ser apuntada como evidencia de la subordinación ideológica de la dictadura de Vargas al fascismo europeo, una vez que la matriz ideológica era la misma, en el sentido de que ambos los regímenes se fundamentaron a partir de una visión autoritaria de gobierno y de organización de sociedad.

En el Estado Novo, el poder personal de Vargas se consolidó mediante estrategias que visaban la personificación del mito, con la ayuda del Estado. El control de los medios de información a través del Departamento de Imprensa e Propaganda (DIP), creado en 1939, estimulaba el culto al Estado y diseminaba rituales colectivos que exaltaban siempre la figura de Vargas. Eran organizados desfiles, manifestaciones y programas de radio que se encargaban de conmemorar el aniversario del dictador, en 19 de abril, a modo de enaltecer sus cualidades personales ${ }^{2}$ y garantizar la diseminación social a través de dispositivos simbólicos de manipulación y coacción, mediados por el reconocimiento tácito de la autoridad que la persona-albo identifica quien a ejerce como pudiendo ejercerla de derecho. ${ }^{3}$

Además, la propaganda del régimen y la represión a sus opositores serian dos facetas del Estado Novo mucho bien representadas por el DIP. Creado para difundir la ideología del Estado Novo junto a las camadas populares y, a partir del ideario autoritario del régimen, contribuir para la construcción de la identidad nacional, el DIP ejercía también una fuerte censura a los medios de comunicación, suprimiendo eventuales manifestaciones de descontentamiento.
La configuración y la duración de la dictadura del Nuevo Estado de Franco (1939-1975) estaba directamente vinculadas al su nacimiento como consecuencia de una guerra civil (1936-1939), en que Franco fuera victorioso, derrotando la Frente Popular. De igual modo, a asimilación por parte de la Iglesia Católica de la guerra civil española como una cruzada determinó un Estado confesional, con repercusiones significativas sobre las instituciones, las costumbres y las relaciones de género.

La Iglesia Católica en su caracterización más conservadora fue uno de los suportes ideológicos fundamentales del régimen franquista, configurando un cuerpo de ideas denominado Nacionalcatolicismo. Las características fundamentales de esa nueva postura eran: la identificación de la idea de patria con la de confesión religiosa; el combate a los valores del mundo moderno, especialmente los democráticos y liberales y la unión de la sociedad política con la sociedad eclesiástica. ${ }^{4}$

En ese contexto, la mujer restituida a su destino biológico de esposa y madre ejemplar, aparece como elemento de agregación entre la sociedad, la familia y el Estado ${ }^{4}$. Así, su confinamiento en el espacio privado asume el sentido de una repartición necesaria de los papeles masculinos y femeninos, en lo que concierne a los deberes de cada uno como patriotas.

El discurso de reclusión de la mujer en el ámbito del hogar, su exclusión del trabajo extradoméstico y de los foros de vida pública tenía una base biológica que se apoyaba en las diferencias congénitas entre el hombre y la mujer, una vez que tales diferencias centradas principalmente en la maternidad era utilizada para legitimar su exclusión de la área económica, social y jurídica, en el régimen franquista. ${ }^{5}$

El discurso franquista dirigido a mujer como reproductora de personas y de tradiciones fuera el contrapunto de una vida de sumisión y de confinamiento en el hogar, reafirmado mediante la limitación jurídica de su capacidad y del control de su cuerpo y actitudes, una vez que fueron instituidas medidas legales que regulaban la segregación de la mujer de la vida pública, a través de una política de represión al cuerpo femenino, mediante la condenación del aborto, la prohibición de métodos contraceptivos y la extirpación de cualquier información sexual pública o privada. ${ }^{5}$

En el caso brasileño, la Constitución de 1934 aseguró a la mujer embarazada el descanso antes y después del parto; prohibió a las mujeres las horas extras, el trabajo nocturno y el trabajo en 
lugares insalubres para que ellas pudiesen tener hijos saludables. También la Carta Constitucional de 1937 mantuvo el mismo principio, cuando incentivó a través de compensaciones, la formación de familias numerosas. Además de eso, en 1941, fue prohibida la propaganda de proceso, sustancia u objeto destinado a provocar aborto. ${ }^{6}$

Esa política a favor de la natalidad tenía el apoyo de varios sectores de la sociedad: interesaba al gobierno brasileño, que tenía el objetivo de implementar el desenvolvimiento económico, basado en la creación de industrias nacionales y también aumentar su base de apoyo en las masas populares; interesaba al Ejército para aumentar su fuerza y promover la seguridad nacional, mediante la ocupación de espacios vacíos del territorio; interesaba a la Iglesia, tanto del punto doctrinario como por raciones políticas, o sea, hacer de Brasil el mayor país cristiano del mundo. ${ }^{7}$

En el caso español, la defensa de la maternidad, consubstanciada por la limitación de la participación femenina en el trabajo extra doméstico respondía también a factores socioeconómicos, pues la sociedad española se presentaba con una economía depauperada y un bajo índice poblacional, en consecuencia de la guerra civil española. El objetivo de cuarenta millones de españoles proclamado por Franco se convierte en una exaltación de crecimiento demográfico como demostración de poder político e de desenvolvimiento de una nación. ${ }^{4}$

De ese modo, la glorificación de la maternidad y la exaltación de atributos morales (abnegación, dulzura) intrínsecos a la naturaleza femenina al tiempo en que reafirmó las diferencias sexuales, de modo a justificar el confinamiento de la mujer en el espacio privado, también legitimó su acceso a la esfera pública, a través de profesiones femeninas, como enfermeras o profesoras, cuya naturaleza del trabajo demandaba la necesidad de cualidades propias a la naturaleza femenina. De ese modo, el trabajo femenino, en el contexto de las dictaduras de Vargas y de Franco se configuraba a través de servicios y ocupaciones adecuadas a la feminidad.

Así, para las enfermeras en Brasil y en España, el incentivo a la natalidad y la necesidad de cualidades tradicionalmente femeninas para el ejercicio de la profesión de enfermera sirvió como moneda de cambio para la inserción de la mujer en espacios tradicionalmente masculinos, lo cual promovió cambios significativos, principalmente relativas a asistencia a la maternidad.
Así, de acuerdo con esa problemática fueron elaborados los siguientes objetivos:

Describir las características histórico-sociales de Brasil y da España en el contexto de los regímenes dictatoriales instaurados por Vargas y Franco

Analizar las representaciones sobre la maternidad en el contexto de eses regímenes y sus implicaciones para la enfermería.

La relevancia de un estudio de dos realidades, Brasil y España, en el que respecta a las relaciones de género, evidenció semejanzas y diferencias en el trabajo de reproducciones de relaciones de poder que reflejaba las construcciones simbólicas, más tradicionales, relativas a las diferencias entre los sexos, una vez que las oposiciones entre el masculino y el femenino son aseguradas por un sistema mítico-ritual que es continuamente legitimado a través de tratamientos diferenciados destinados a los hombres y las mujeres, pues los rituales consagran y legitiman las diferencias, es decir, instituí las diferencias al tratar de forma distinta los hombres y las mujeres.

\section{METODOLOGÍA}

Estudio histórico-social, derivado del proyecto de investigación titulado "La Trayectoria de la Enfermería en regímenes dictatoriales: un estudio comparado Brasil/España", apoyado por la "Coordenação de Aperfeiçoamento de Pessoal de Nível Superior (Capes)" y vinculado al GIR "Grupo de Investigación de Historia de Género y de las Mujeres Leticia Valle" (Universidad de Valladolid - España).

La recogida de datos fue realizada en el período de 13 de mayo a 29 de agosto de 2008. De acuerdo con el preconizado por el método histórico, lo estudio ha utilizado fuentes primarias y secundarias. Las primarias constó de documentos escritos como constituciones, leyes y decretos brasileños y españoles; noticias publicadas en el periódico "El Norte de Castilla", relativas al período de 1939-1975, archivadas en la Biblioteca Pública de Valladolid, además de discursos y ponencias localizadas en el Archivo General de La Guerra Civil, de Salamanca. Las fuentes secundarias se refieren a la literatura sobre cuestiones de género para la enfermería en el contexto de las dictaduras de Vargas, en Brasil, y de Franco, en España.

El análisis de datos comportó las etapas de organización y clasificación de las fuentes primarias elegidas. Para la interpretación se ha utilizado 
la Teoría do Mundo Social del sociólogo francés Pierre Bourdieu, especialmente en lo que concierne a los conceptos de campo, poder simbólico y dominación masculina. ${ }^{3} \mathrm{La}$ aplicación de eses conceptos a los resultados de la investigación consubstanció el análisis y discusión sobre las estrategias de lucha simbólica emprendidas por las mujeres por visibilidad y sus conquistas en los espacios público y privado. De esa forma, las marcas de las posiciones sociales y los signos exteriores que distinguen esas posiciones fueron temas abordados en la lectura del corpus documental.

\section{RESULTADOS Y DISCUSIÓN}

\section{Las mujeres e las dictaduras de Vargas y de Franco}

El franquismo y el varguismo convergen en el sentido de criticar a la democracia parlamentar, a la pluralidad de partidos y la representación autónoma de intereses. De ese modo, la condenación del modelo republicano anterior fue acompañada de su estigmatización, bajo acusación de tener determinado la pérdida de valores tradicionales, entre eles la familia y el hogar, tema clave para definir la dicotomía nuevo orden versus republicano. ${ }^{4}$ La recuperación del sentido cristiano de la familia, fundado en la recuperación del papel femenino tradicional, fue un objetivo compartido por los diferentes componentes del régimen.

En el caso brasileño, en el comando del Estado, el poder personal de Getúlio Vargas representaba la instancia decisiva en las resoluciones fundamentales. Para llegar a esas resoluciones el oía un círculo formado por amigos íntimos, miembros de Casa Civil y de Casa Militar y ministros de Estado. Getúlio mantenía relaciones próximas no con el ministerio como un cuerpo colectivo, mas con cada uno de sus elementos. ${ }^{1}$

En lo que respecta al aspecto socioeconómico, el Estado Novo se configuró como una alianza de la burocracia civil y de la burguesía militar, cuyo objetivo común inmediato era el de promover la industrialización del país, sin problemas sociales. En ese sentido, la burocracia civil defendía el programa de industrialización por considerar necesaria la verdadera independencia del país; los militares por acreditaren que la instalación de una industria de base fortalecería la economía, por eles considerada componente importante de la seguridad nacional y los industriales por estaren convencidos de que el incentivo a la industrialización dependía de la acción interventora del Estado. ${ }^{1}$
El creciente interese de Vargas en promover la industrialización del país tuvo reflejos en el campo educacional. Aunque Gustavo Capanema (Ministro de la Educación y Salud de Getúlio Vargas de 1934 a 1945) tenga promovido una reforma de la enseñanza secundaria (Decreto-Ley N. 4.244, de 9 de abril de 1942), la organización de la enseñanza industrial también fue tema de su preocupación, pues estableció las bases de la preparación profesional cualificada. ${ }^{8}$

En lo que respecta a la condición de la mujer, la Ley Orgánica de Enseñanza Secundaria, también conocida como Reforma Capanema, que permanecería en vigor hasta la aprobación de la Ley de Directrices y Bases de la Educación Nacional, en 1961, es ejemplar en el sentido de reproducir y legitimar la división jerarquizada de los papeles sociales del hombre y de la mujer, en lo que concierne a las especificidades de los programas de enseñanza, pues la orientación metodológica de los programas tuvo en observación la naturaleza de la personalidad femenina y la misión de la mujer dentro del hogar. ${ }^{9}$

Esa misma ley determina que la enseñanza secundaria de las mujeres se realice en clases exclusivamente femeninas (Artigo 25, Ítem 1), dificultando la relación entre los sexos. Además, la prescripción de la asignatura Economía Doméstica, únicamente para las mujeres (Artigo 24, Ítem 3) ratifica la repartición de los papeles sexuales a través de diferencias inherentes a la formación del hombre y de la mujer, a las cuales reproducen la dominación masculina que se inscribe en la naturaleza y distribución de las actividades o en los rituales colectivos o privados, mediante las conductas de marginalización impostas a las mujeres a través de su exclusión de lugares públicos y, por consiguiente de exclusión de tareas más nobles. ${ }^{10}$

En el caso español, la coeducación también es suprimida como una demostración de pérdida de valores que representaba la República. También la asignatura denominada Enseñanzas del Hogar solamente para las clases femeninas evidencia los principios antagónicos de la identidad femenina y masculina, implícitos en la exclusión de las mujeres de tareas más importantes. Además, la educación femenina además de la enseñanza primaria era considerada superflua y sólo se admitía la formación universitaria en profesiones tradicionalmente ligadas a la mujer o la formación en Letras para aquellas que pertenecían a una clase alta, tiendo en vista la necesidad de tener una variedad de asuntos para ofrecer al su futuro marido. ${ }^{5}$ 
En ese sentido, el ideal de emancipación de la mujer en el régimen franquista estaba totalmente aniquilado, siendo el hogar el lugar de la mujer. Tanto así que, el Fuero del Trabajo, de 9 de marzo de 1938 determinó una serie de medidas en consonancia con la imagen y el papel social de la mujer en la nueva España, prohibiendo el trabajo a la mujer casada, además de divulgar una lista de trabajos vetados a las mujeres. Como medidas mitigadoras, el Fuero del Trabajo previa subsidios $\mathrm{y}$ ayudas a las familias numerosas y premios de natalidad. ${ }^{4}$

Además de eses dispositivos legales, la Iglesia Católica apoyó ostensivamente la exaltación de la familia y la vuelta de la mujer al mundo doméstico, pues en el caso específico de las mujeres, la moral sexual y familiar de la Iglesia Católica, extremamente tradicional y antifeminista se identificaba perfectamente con el nuevo régimen político español. ${ }^{4}$

El trecho trascrito del discurso hecho por Serrano Suñer (Ministro y Máximo Dirigente de Partido Único, Falange Española Tradicionalista y de las Juntas de Ofensiva Nacional Sindicalista (JONS), en la Sesión de Clausura del Consejo Nacional de la Sección Femenina de Falange, en 1940) evidencia el papel social de la mujer en el contexto del nuevo régimen: "[...] la misión de la mujer está en hogar y su labor en los problemas que con él de relacionan, señalándolas allí como medio mejor y más eficaz de servir a la Patria". 11:2 No mismo discurso, Serrano Suñer expresa la importancia de la alianza del Estado con la Iglesia en ese proceso de socialización de la mujer: “[...] En esta tarea encomendada a la mujer ha señalado un auxiliar precioso y insubstituible: la iglesia que dentro de su misión prestará con ello a la Patria un servicio inapreciable. Porque, efectivamente, nada mejor que la doctrina de la Iglesia para llevar a cabo ese apostolado que la mujer si le encomienda $[\ldots]^{\prime \prime} .11: 2$

En ese sentido, a la Iglesia Católica es conferida la función de reelaboración y mantenimiento del orden social vigente, mediante su autoridad religiosa, con vistas a combatir las tentativas heréticas de subversión de ese orden, pues las doctrinas constituyen no solamente modalidades simbólicas de transfiguración de la realidad social, una vez que ordenan, clasifican, sistematizan y representan el mundo social. ${ }^{12}$ De esa forma, el control de la moral femenina no se dio únicamente en el plan eclesiástico o a través de vivencias religiosas relativas a la práctica cotidiana de la religión (misas, procesiones, novenas, entre otras), más también mediante un proceso de control de los cuerpos y de la sexualidad.

La ponencia de de Don Santos Begueristáin, promovida por la Asociación Católica de Padres de Familia, en 1943, consagra simbólicamente la asimetría de género, al destacar, en el hombre y en la mujer los signos exteriores más conformes a la definición social de su diferenciación sexual, mediante el estímulo de prácticas convenientes a cada sexo: "[...] afortunadamente en España está arraigada la idea del matrimonio no sentido cristiano. El hombre tiene sus hijos, su esposa, considera esencial la fidelidad, defiende como un tesoro inapreciable la virtud de sus hijos y el honor de sus hijas" ${ }^{13: 2}$

Esa regularización de orden física y social coloca el hombre en la condición de señor del privado y de la familia que ellos gobiernan y representan, delegando a las mujeres la gestión del cotidiano, ${ }^{14}$ constituyendo las mujeres como subordinadas y sometidas en consonancia con la jerarquía entre los sexos, proclamada como necesaria a la ordenación de los espacios sociales en la sociedad brasileña y española, en el contexto de las dictaduras de Vargas y de Franco.

\section{La mujer y la enfermería en las dictaduras de Vargas y de Franco}

En Brasil y en España, la maternidad y la moral femenina, fundamentos del orden social, consagraban la necesidad de reclusión de la mujer en el espacio doméstico. La moralidad femenina, controlada por la Iglesia, se traducía en la indumentaria o en pautas de comportamiento que tendría como referentes la pureza y la decencia moral $^{5}$. El trecho trascrito del discurso del Papa, en la Sesión de Clausura del Primero Congreso de Asociaciones Católicas de Trabajadoras Italianas sacraliza los principios antagónicos de la identidad masculina y femenina, mediante la distinción de los papeles de cada uno en la sociedad: "La mujer es el corazón de la familia y el cuidado de la casa, en la cual ella reina, constituyen centro y campo de su actividad $[\ldots]^{\prime \prime} .{ }^{15: 1}$

Además, la naturaleza antintelectual femenina también se configura como uno de los argumentos que respaldaban tanto la exclusión del trabajo como el retorno al hogar. José María Pemán (entonces director de Real Academia Española de Lengua) trata el tema en las ponencias tituladas "De doce cualidades de la mujer", don- 
de reafirma el dualismo masculino y femenino, mediante la afirmación de que las deficiencias intelectuales de la mujer son compensadas por sus atributos típicamente femeninos: "Dios hace las cosas bien y con orden. ¿Para qué iba a construir con excesivo mecanismo intelectual el alma de un ser destinado, por esencia, a las bellas sinrazones del cariño?". ${ }^{16: 387}$

De modo coherente con el discurso de la Iglesia y la ideología del régimen, la afirmación de Pilar Primo de Rivera (Delegada Nacional de La Sección Femenina de Falange Española Tradicionalista y de las JONS), en 1942, consagra elementos de la ideología totalitaria en el que concierne a la inferioridad de la mujer en relación al hombre: "Las mujeres nunca descubren nada; les falta, desde luego el talento creador, reservado por dios para inteligencias varoniles". 17:2

Además, el proceso de socialización de la mujer, emblema de la nueva España, contempla elementos que sacralizan la dominación masculina, describiéndola como invariable y eterna. El trecho trascrito de la revista Medina, de 13 de agosto de 1944 (revista de la Sección Femenina dirigida al público femenino) evidencia la incorporación de las relaciones de poder que se expresan en las oposiciones que fundamentan el orden simbólica: "La vida de toda mujer, a pesar de cuanto ella quiera simular - o disimular- no es más que un eterno deseo de encontrar a quien someterse...". 17:3

Sin embargo, en el contexto de las dictaduras de Vargas y de Franco, la propaganda del régimen evocaba la mujer como un ser superior en cierta medida al hombre, por sus virtudes físicas (la maternidad) y sus atributos morales (abnegación, dulzura) frente a un hombre siempre más grosero y guerrero. ${ }^{5}$ Subyacente a la conversión de la mujer en receptáculo de amor y de vida, tal conversión legitimaba una relación de dominación, inscribiéndola en una naturaleza biológica que es, por su vez, "una construcción de géneros como dos esencias sociales jerarquizadas", ${ }^{10: 33}$ que destacaba, en el hombre y en la mujer, los signos exteriores relativos a la distinción sexual, a modo de estimular las prácticas convenientes al sexo, contribuyendo para la invisibilidad de la mujer en los espacios públicos, habitualmente consagrados a los hombres. ${ }^{18}$

En lo que respecta al parto y al nacimiento, desde la Edad Media, la doctrina de la Iglesia enfatizaba la noción de impureza. Esa noción que estaba asociada a la pérdida de sangre, orina y heces en el momento de expulsión del niño, de- terminó la creación de normas rígidas relativas al parto y puerperio, bien como la valorización de la dolor con el objetivo de purgación del pecado de la mujer. ${ }^{19}$

La arte de hacer el parto fue durante muchos años constituida como un saber femenino, rico en conocimientos populares, orientado por la pela intuición y por la experiencia del cotidiano. Entretanto, en el contexto de hospitalización de la sociedad, en el Brasil y España, esa práctica fue transformada en saber masculino, repleto de conocimientos biológicos y tecnológicos, conformes con a la ciencia racional. ${ }^{20}$

En Brasil, en el inicio de la colonización, el grupo de mujeres que hacían el parto era constituido por negras, portuguesas y caboclas que eran designadas como curiosas. Además de cuidar del parto esas mujeres eran acusadas de hacer hechicería, provocar aborto y cometer infanticidio. ${ }^{21}$ Pocas fueron las parteras formadas por escuelas de partos. Tanto así que, en el período de 1833-1876, solamente diez alumnas concluirán el curso de partos de Facultad de Medicina del Rio de Janeiro y el de Bahia fue también poco frecuentado. ${ }^{22}$

Durante mucho tiempo, la participación del médico en el parto era vista como algo degradante, pues la partera cuidaba de secreciones y olores provenientes del cuerpo femenino, lo que explicaba el número mayor de negras haciendo el parto: "se la sangre de una mujer blanca era vista con repugnancia, ¿cómo no sería la de una mujer negra y esclava, o mestiza y pobre?". ${ }^{21: 40}$

Entretanto, los médicos empeñados en establecer su hegemonía en el campo de la salud empezaron a disputar las mujeres embarazadas con las parteras, ${ }^{22}$ en el ámbito de un creciente proceso de uso de medicinas para combatir las enfermedades, al largo del siglo XX. En el caso brasileño, las enfermeras diplomadas, aliadas a la Iglesia Católica, al defender el parto hospitalario lo hacían en provecho propio, porque este era el lugar que ella se encontraba mejor posicionada, aunque con menos poderes que el médico, mas con más poderes que la partera. ${ }^{20}$ Así, los médicos garantizaron el control de la práctica de enfermería y el apoyo para eliminar las parteras, reproduciendo con eufemismo y disimulación, las diferencias y jerarquías existentes.

La enfermera, al defender el hospital como local de realización del parto, reproducía el discurso de la Iglesia Católica que, ante los temores de la realización de abortos por parteras o médicos en sus consultorios $\mathrm{y}$, también para controlar la 
sexualidad femenina, era contraria a la realización del parto fuera del hospital. ${ }^{20}$ En ese contexto, las enfermeras no realizaban el parto, mas incorporaran las habilidades complementares o auxiliares del trabajo médico, o sea, los cuidados de higiene, conforto y cumplimento de ordenes médicas.

Así, la posición de las enfermeras en el campo obstétrico reproducía las estructuras de poder y dominación social relativas a la mujer, en la sociedad de la época, una vez que la dominación simbólica se respalda en el reconocimiento de los principios en nombre de los cuales ella se ejerce, ${ }^{23}$ en ese caso expresado por el reconocimiento por parte de las enfermeras y parteras, del saber del médico en el campo de la obstetricia. En lo que concierne al cuerpo de la mujer, entre los siglos XVII e XIX, fueron creados dispositivos de dominación del cuerpo femenino, mediante la denominación de nombres de médicos a las características anatómicas y fisiológicas del cuerpo de la mujer embarazada (Tubérculo de Montegomery, Señal de Jacquemier Kluge, etc.). Simbólicamente, la reproducción de esas denominaciones constituí las mujeres como objetos simbólicos de la dominación masculina.

En lo que respecta la posición de la mujer durante el parto (posición denominada ginecológica) con los médicos a sus pies, el (el médico) observa la vagina como algo disociado de la persona y, por lo tanto, reducida a la condición de cosa, a modo de neutralizar simbólicamente todas las connotaciones potencialmente sexuales de tal procedimiento, pues el cuerpo de la mujer es objeto de atención por ser considerado un peligro y una amenaza.

También la interdicción del uso de fórceps (instrumento que se utiliza para facilitar la salida de la cabeza del bebé del canal de parto) a la partera y su utilización por los médicos parteros, contribuyo para reforzar las relaciones de dominación masculina, debido la representación simbólica de un instrumento que penetraba en el cuerpo de la mujer ${ }^{20}$ pues, el acto sexual, en la percepción de Bourdieu es concebido por los hombres como una forma de dominación y de posesión, una vez que expresa el principio de división fundamental entre el masculino, activo, y el femenino, pasivo. ${ }^{10}$

En lo que concierne a enfermería española, después de la guerra civil, la primera norma que afecta las profesiones sanitarias es la de 1 de marzo de 1940, la cual estableció que los estudios de matrona deberían realizarse preferentemente en la Casa de Salud Santa Cristina, en Madrid. Los estudios de Matrona contemplaban los mismos requisitos para obtención del título de practicante, mientras que para las matronas era necesario ser mayor de edad y tener autorización del marido, cuando casada, ${ }^{24}$ lo que evidencia tratarse de una categoría eminentemente femenina.

Ese dato gana más expresividad cuando se observa las funciones de las enfermeras y de las matronas, especificadas en la Ley de 1945 que establecía las competencias sanitarias dos tres grupos sanitarios existentes (enfermeras, practicantes y matronas). La ley establecía que las matronas y enfermeras eran profesiones exclusivamente femeninas. Las matronas deberían atender los partos y auxiliar al médico especialista en la asistencia a las mujeres embarazadas, bajo sus órdenes. En el caso de las enfermeras, sus funciones se centraban en un fuerte carácter técnico y vocacional, también con una significativa dependencia del médico y con connotaciones exclusivamente femeninas de la profesión. ${ }^{24}$

De ese modo, la tarea de cuidar era conjugada en el femenino y, por lo tanto, se mostraba como una práctica sexuada, implicando en la necesidad de atributos que no se encuadraban en el orden de los conocimientos técnicos y científicos, más en las cualidades percibidas como naturales a las mujeres. El trecho abajo, sacado de una correspondencia encaminada por un médico, entonces director de un español, a la Delegación Provincial de la Sección Femenina sobre el desempeño profesional de una enfermera ratifica esta afirmación: "demostró todo el tiempo una gran lealtad al mando y observando siempre ejemplarismo en su conducta, constituyendo su actuación modelo de abnegación y espíritu de sacrificio".*

\section{CONSIDERACIONES FINALES}

La importancia atribuida a la maternidad, en el contexto favorable a la natalidad en las sociedades brasileña y española, determinó repercusiones significativas inherentes a la profesionalización de la mujer y de la enfermera, una vez que legitimó su inserción honorable en el espacio público, a modo de reproducir en ese mismo espacio las cualidades femeninas necesarias al cuidado.

Sin embargo, en ese mismo espacio, donde el femenino es ocultado simbólicamente, inclusive en el cuidado realizado por mujeres que, ni siempre tienen en cuenta de que reproducen la dominación masculina, mediante la aceptación tácita de sus interdicciones en ese espacio, producto de la incorporación de las relaciones de poder.

\footnotetext{
* Archivo Provincial de Valladolid, Caja, 1027.
} 
El trabajo de reproducción de esas relaciones de poder, en el contexto de las dictaduras de Franco y Vargas, fue garantizado por tres instancias principales: la familia, la Iglesia y el Estado, que, objetivamente orquestados, convergían en el sentido de emprendieren estrategias que naturalizaban esas reproducciones, mediante la consagración de la maternidad y de la mujer como reproductora de las tradiciones.

\section{REFERENCIAS}

1. Fausto B. História do Brasil. São Paulo (SP): Edusp; 1996.

2. Camargo A. carisma e personalidade: da conciliação ao maquiavelismo. In: D'araújo MC, organizador. As instituições brasileiras da Era Vargas. Rio de Janeiro (RJ): FGV; 1999. p.17.

3. Bourdieu P. A economia das trocas lingüísticas: o que falar quer dizer. São Paulo (SP): Edusp; 1998. p.95.

4. Gomes-Ferrer G, Cano G, Barrancos D, Lavrin A. Historia de las mujeres en España y América Latina: del siglo XX a los umbrales del XXI. Madrid (ES): Ediciones Cátedra; 2006. p.267.

5. Gracía J, Ruiz GMA. La España de Franco (1939-1975). España(ES): Ed Editorial Síntesis; 2004.

6. Brasil. Decreto-Lei No 3.689, de 03 de outubro de 1941: dispõe sobre o Código de Processo Penal. Diário Oficial da República Federativa do Brasil, 13 out 1942. Seção 1.

7. Mattos CM. Brasil: geopolítica e destino. Rio de Janeiro (RJ): Ed José Olympio; 1979. p.57-60.

8. Brasil. Decreto-Lei No 4.073, de 30 de janeiro de 1942: dispõe sobre a Lei Orgânica do Ensino Industrial. Diário Oficial da República Federativa do Brasil, 25 Fev 1947. Seção 2.

9. Brasil. Lei No 4.024, de 20 de dezembro de 1942: dispõe sobre Fixa as Diretrizes e bases da Educação Nacional. In: Ministério da saúde. Fundação de Serviços de saúde Pública. Enfermagem, legislação e assuntos correlatos. $3^{\text {a }}$ ed. Rio de Janeiro: SESP, 1974. v 2, p 227-36.

10. Bourdieu P. A dominação masculina. Rio de Janeiro (RJ): Ed Bertrand do Brasil; 1999.
11. Hernandez M. El discurso del señor serrano Suñer y la misión de la mujer española. Periódico El Norte de Castilla, 1940 enero 21; Seção 1 (col 1).

12. Bourdieu P. A economia das trocas simbólicas. São Paulo (SP): Editora Perspectiva; 2001.

13. Begueristáin DS. La misión de la mujer española. Periódico El Norte de Castilla, 1943 enero 09; Seção1 (col. 2)

14. Perrot M. Minha história de mulheres. São Paulo (SP): Editora Contexto; 2007.

15. Hernandez M. El papa se dirige a 6000 mujeres. Periódico El Norte de Castilla, 1945 agosto 18; Seção1 (col. 4)

16. Pemán JM. De doce cualidades de la mujer. Madrid (ES): Ed Alcor; 1947.

17. Rivera PP. Perfecta mujer nacional-católica. Rev. Medina.1944 Jun; 5(1):1-5.

18. Santos TCF, Barreira IA. A mulher e a enfermeira na nova ordem social do Estado Novo. Texto Contexto Enferm. 2008 Jul-Dez;17(3):587-93.

19. Tanaka ACD, Alvarenga AT. Tecnologia e medicalização na concepção e anticoncepção. In: Loren G, Juan D, organizadores. Saúde social e reprodutiva no Brasil. São Paulo (SP): Hucitec; 1999. p.201.

20. Progianti JM. Parteiros, médicos e enfermeiras: a disputada arte de partejar (Rio de Janeiro, 1934/1951) [tese]. Rio de Janeiro (RJ): Universidade Federal do Rio de Janeiro. Programa de Pós-Graduação em Enfermagem da Escola de Enfermagem Anna Nery; 2001.

21. Mott ML. Parteiras no século XIX: Mme. Durochere sua época. In: Costa AO, Bruschini C, organizadores. Entre a virtude e o pecado. Rio de Janeiro (RJ): Ed Rosa dos Tempos, Fundação Carlos Chagas; 1992. p.40-1.

22. Osawa RH, Riesgo MLG, Tsunechiro MA. Parteirasenfermeiras e enfermeiras-parteiras: a interface de profissões afins, porém distintas. Rev Bras Enferm. 2006; 59 (5):699-702.

23. Bourdieu P. Razões e práticas. São Paulo (SP): Papirus; 1996.

24. Magdalena STP. Historia de la Enfermería. In: Carmen FF, Margarita GA, Magdalena STP, María DSP, organizadores. Enfermería fundamental. Barcelona (ES): Ed Masson; 1995.

E-mail: taniacristinafsc@terra.com.br 